\title{
Immunohistochemical profile of cytokines and growth factors expressed in vestibular schwannoma and in normal vestibular nerve tissue
}

\author{
SAMANTA TAURONE $^{1 *}$, ENRICA BIANCHI $^{1 *}$, GIUSEPPE ATTANASIO $^{1}$, CIRA DI GIOIA $^{2}$, \\ ROCCO IERINÓ ${ }^{2}$, CECILIA CARUBBI $^{3}$, DANIELA GALLI ${ }^{3}$, FRANCESCO SAVERIO PASTORE $^{4}$, \\ FELICE GIANGASPERO $^{2,5}$, ROBERTO FILIPO ${ }^{1}$, CHRISTIAN ZANZA ${ }^{1}$ and MARCO ARTICO ${ }^{1}$
}

\begin{abstract}
Departments of ${ }^{1}$ Sensory Organs and ${ }^{2}$ Radiology, Oncology and Human Pathology, Sapienza University of Rome, Rome I-00161; ${ }^{3}$ Department of Biomedical, Biotechnological and Translational Sciences, University of Parma, Parma, Emilia-Romagna I-43126; ${ }^{4}$ Division of Neurosurgery, Tor Vergata University of Rome, Rome I-00173; ${ }^{5}$ Neuromed Institute, Pozzilli, Isernia I-86077, Italy
\end{abstract}

Received December 5, 2013; Accepted January 21, 2015

DOI: $10.3892 / \mathrm{mmr} .2015 .3415$

\begin{abstract}
Vestibular schwannomas, also known as acoustic neuromas, are benign tumors, which originate from myelin-forming Schwann cells. They develop in the vestibular branch of the eighth cranial nerve in the internal auditory canal or cerebellopontine angle. The clinical progression of the condition involves slow and progressive growth, eventually resulting in brainstem compression. The objective of the present study was to investigate the expression level and the localization of the pro-inflammatory cytokines, transforming growth factor- $\beta 1$ (TGF- $\beta 1$ ) interleukin (IL)-1 $\beta$, IL- 6 and tumor necrosis factor- $\alpha$ (TNF- $\alpha$ ), as well as the adhesion molecules, intracellular adhesion molecule-1 and vascular endothelial growth factor (VEGF), in order to determine whether these factors are involved in the transformation and development of human vestibular schwannoma. The present study investigated whether changes in inflammation are involved in tumor growth and if so, the mechanisms underlying this process. The results of the current study demonstrated that pro-inflammatory cytokines, including TGF- $\beta 1$, IL- $1 \beta$ and IL- 6 exhibited increased expression in human vestibular schwannoma tissue compared with normal vestibular nerve samples. TNF- $\alpha$ was weakly expressed in Schwann cells, confirming that a lower level of this cytokine is involved in the proliferation of Schwann
\end{abstract}

Correspondence to: Professor Marco Artico, Department of Sensory Organs, Sapienza University of Rome, 155 Viale del Policlinico, Lazio, Rome I-00161, Italy

E-mail: marco.artico@uniroma1.it

*Contributed equally

Key words: Schwann cells, acoustic neuromas, transforming growth factor- $\beta 1$, interleukin- $1 \beta$, tumor necrosis factor- $\alpha$, interleukin-6, vascular endothelial growth factor, intracellular adhesion molecule-1 cells. Neoplastic Schwann cells produce pro-inflammatory cytokines that may act in an autocrine manner, stimulating cellular proliferation. In addition, the increased expression of VEGF in vestibular schwannoma compared with that in normal vestibular nerve tissue, suggests that this factor may induce neoplastic growth via the promotion of angiogenesis. The present findings suggest that inflammation may promote angiogenesis and consequently contribute to tumor progression. In conclusion, the results of the present study indicated that VEGF and pro-inflammatory cytokines may be potential therapeutic targets in vestibular schwannoma. Further studies are necessary to confirm the involvement of these factors in the growth of neoplasms and to develop inhibitors of pro-inflammatory cytokines as a potential treatment option in the future.

\section{Introduction}

Vestibular schwannomas (VS), also known as acoustic neuromas, are the most frequent benign tumor of the lateral skull base, and originate from Schwann cells of the vestibular branch of the eighth cranial nerve. VSs are neoplasms that occur as a result of the increased proliferation of Schwann cells and are diagnosed histopathologically by the presence of singular architectural patterns called Antoni A and Antoni B areas. VS represents $8 \%$ of all primary intracranial tumors (1). Individuals between 30 and 60 years old are the most frequently affected, and there is no gender prevalence $(2,3)$. The majority of VSs are sporadic and, in general, are benign slow-growing neoplasms. They exhibit a wide variability in growth rate and size. If growth continues it may result in complex pathological conditions, including brainstem compression and hydrocephalus. Magnetic resonance imaging has a central role in the diagnosis of this condition, and complete surgical resection remains the preferred treatment. The growth of VS is not directly correlated with tumor size, symptoms, duration of symptoms or the patient's age (4). The growth rate of VS is heterogeneous, at 0.3-1.42 mm/year. VS evolves from an abnormal growth and proliferation of Schwann 
cells, at their junction with glial cells surrounding the vestibular nerve. This neoplasm is exclusively formed from the hyperproliferation of Schwann cells and associated neovasculature $(5,6)$. Schwann cells are principal glial cells of the peripheral nervous system. During embryogenesis, they migrate along axons and synthesize a basal lamina, consisting predominantly of laminin, collagen and proteoglycans. Schwann cells have an important role in nerve regeneration following laceration, when they replace damaged Schwann cells and synthesize a new basal lamina and myelin sheath. Following nerve injury, successful remyelination of damaged axons by Schwann cells relies upon a combination of signals that Schwann cells receive from demyelinated axons during in the inflammatory response. These signals initially prompt Schwann cells to re-enter the cell cycle and subsequently to differentiate into myelinating cells (6). Axonal myelination is important for the functional recovery of injured peripheral nerves. In particular, it facilitates rapid saltatory impulse conduction by producing a faster conduction velocity of action potentials $(7,8)$. In order to better understand the molecular mechanisms of Schwann cells, including cell proliferation, migration, survival and apoptosis during peripheral nerve injury, the present study aimed to invetigate the involvement of certain inflammatory cytokines and growth factors in VS. The majority of the pathogenetic mechanisms regulating neoplastic growth in vestibular nerve cells, remain to be elucidated. A number of studies have demonstrated that neurotrophins and growth factors have a role in governing the development of homeostasis, cell survival and regeneration processes within Schwann cells $(9,10)$. Although further neoplastic growth appears to depend on cytokines with angiogenic and mitogenic properties, data concerning the involvement of growth factors in VS growth are not currently available. Trophic factors, including transforming growth factor- $\beta 1$ (TGF- $\beta 1$ ) and vascular endothelial growth factor (VEGF), have been designated as possible key mediators of VS growth. TGFs are a family of polypeptides involved in wound healing and tumorigenesis in vivo. TGF- $\beta 1$ may act as either an inhibitor or stimulator of cell proliferation, depending on the cell type and growth conditions. TGF- $\beta 1$ may be involved in the development of VS, stimulating the proliferation of Schwann cells. The signalling pathway of TGF- $\beta 1$ is activated by two transmembrane serine/threonine kinases, TGF- $\beta$ R 1 and TGF- $\beta$ R2. The type 2 receptor (TGF- $\beta$ R2) is involved in the antiproliferative activity of TGF- $\beta$, whereas the type 1 receptor (TGF- $\beta$ R1) appears to cause cellular proliferation following cell-matrix interactions (11). Interactions between cytokines and Schwann cells are involved in the development of disorders of the peripheral nervous system. Tumor necrosis factor- $\alpha(\mathrm{TNF}-\alpha)$ is a pro-inflammatory cytokines produced by activated macrophages in response to pathogens and other noxious stimuli. TNF- $\alpha$ is released by Schwann cells as well as by macrophages (12). TNF- $\alpha$, one of the major initiators of the inflammatory cascade, activates pleiotropic functions in physiological and pathological conditions by binding to its receptors, type I (TNFRI) and type II (TNFRII). Wang et al (13) described the involvement of TNF- $\alpha$-associated signalling molecules, including a baculoviral inhibitor of apoptosis repeat-containing protein (BIRC) 2, BIRC 3 and TNFRI, in the anti-apoptotic process of injured peripheral nerves, indicating that a higher level of TNF- $\alpha$ may induce apoptosis in Schwann cells in vitro, while a lower level of TNF- $\alpha$ may not act in the same way.
Wagner and Myers (12) confirmed that the production of TNF- $\alpha$ by peripheral nerve glial cells has a pathogenic role in nerve injury. A number of studies have investigated the possible role of interleukin (IL)-6 in peripheral nerve regeneration $(14,15)$. However, the molecular mechanisms underlying the involvement of IL-6 in the development of Schwann cells remain to be fully elucidated. The induction of pro-inflammatory genes by IL-6 in Schwann cells may indicate that IL-6 is involved in the degeneration of injured neurons, in cooperation with other inflammatory cytokines, such as TNF- $\alpha$. Certain pro-inflammatory cytokines, including TNF- $\alpha$, IL- $1 \beta$ and IL- 6 are known to induce the expression of adhesion molecules. Adhesion molecule expression, including that of intracellular adhesion molecule-1 (ICAM-1), is tightly-regulated by cytokines generated during an inflammatory response (16). ICAM-1 is able to facilitate leukocyte attachment and interactions with cells from the target tissue (17). The expansion of any solid tumor with a volume $>2-3 \mathrm{~mm}$ is reliant upon angiogenesis to provide oxygen and nutrients to the enlarging tumor. VEGF induces angiogenesis through endothelial cell proliferation and migration. It is considered to be one of the most potent pro-angiogenic factors, causing vasodilatation, vascular permeability and angiogenesis. Angiogenesis is defined as the process of new blood vessel formation from pre-existing vasculature and involves a cascade of processes, during which the vessel's basal membrane and the surrounding extracellular matrix are modified by endothelial cell proliferation and migration (18). The binding of VEGF to high-affinity receptors, VEGFR-1 and VEGFR-2, promotes extravasation of plasma proteins from tumor vessels, thereby forming a temporary extravascular matrix which favors the migration and proliferation of endothelial cells, resulting in new blood vessel formation (19). The aim of the present study was to investigate the expression of pro-inflammatory cytokines in VS compared with normal vestibular nerve tissue, using immunohistochemistry, in order to improve understanding of the pathogenesis of this disease. An increased knowledge of this subject may have significant clinical consequences in terms of improvement in clinical treatment, prevention of the postsurgical relapse, and a reduction in the severity of inflammation, tissue invasion, tumor proliferation and angiogenesis.

\section{Materials and methods}

Ethical considerations and VS samples. In accordance with approval of the ethical committee of Policlinico Umberto, Sapienza University of Rome (Rome, Italy), tissues were harvested from ten randomly selected patients, four females and six males, with unilateral, sporadic VS that had been removed surgically, and ten healthy control samples, which consisted of three females and seven males with Ménière's syndrome following vestibular neurectomy. The patients, aged between 45 and 69 years, consisted of four females and six males. Routine histopathological examination confirmed the diagnosis of benign VS in the samples, which included one patient with a tumor recurrence.

Prior to signing the consent form, patients were informed about the study in detail and were given sufficient time to ask questions. The study was conducted in accordance with the Declaration of Helsinki. Each clinical unit selected specimens and assigned a number to each sample, followed by a letter 
indicating the participating unit. For each case, a report was prepared, indicating the age and gender of the patient, as well as their clinical signs and symptoms. Control morphological sections were stained with hematoxylin and eosin (H\&E), or processed for immunohistochemistry. The following molecules were investigated in the tumoral samples and in normal vestibular nerve specimens: VEGF, TGF- $\beta 1$, IL- $1 \beta$, IL-6, ICAM-1 and TNF- $\alpha$.

Immunohistochemical analysis. For light microscopic immunohistochemical analysis, small fragments of VS were processed according to the avidin-biotin complex/horseradish peroxidase technique. These samples were washed in phosphate-buffered saline (PBS), fixed in $10 \%$ formalin and embedded in paraffin according to a standard procedure (20). Serial 3- $\mu \mathrm{m}$ sections were cut using a rotative microtome (RM2265; Leica Biosystems, Wetzlar, Germany), mounted on gelatin-coated slides and processed for immunohistochemistry. These sections were deparaffinized in xylene and dehydrated. They were immersed in citrate buffer (pH 6.0; 15M103; BioOptica Milano, S.p.A, Milan, Italy) and subjected to microwave irradiation twice for $5 \mathrm{~min}$. Subsequently, all sections were treated for $30 \mathrm{~min}$ with $0.3 \%$ hydrogen peroxide in methanol in order to quench endogenous peroxidase activity. To block non-specific binding, the slides were incubated in 3\% normal goat serum (S-100; Vector Laboratories Burlingame, CA, USA) in PBS (15M108; BioOptica Milano, S.p.A.) for $30 \mathrm{~min}$ at room temperature. The slides were incubated overnight at $4^{\circ} \mathrm{C}$ with the following antibodies all purchased from Santa Cruz Biotechnology, Inc. (Dallas, TX, USA): Rabbit anti-IL-1 $\beta$ polyclonal antibody (1:50; Sc-7884); rabbit anti-IL-6 polyclonal antibody (1:200; sc-7920); mouse anti-TNF- $\alpha$ monoclonal antibody (1:100; sc-52791); mouse anti-VEGF monoclonal antibody (1:200; sc-152); mouse anti-ICAM-1 monoclonal antibody (1:50; sc-107); and rabbit anti-TGF- $\beta 1$ polyclonal antibody (1:200; sc-146). Optimal antisera dilutions and incubation times were assessed in a series of preliminary experiments. Following exposure to the primary antibodies, slides were rinsed twice in PBS and incubated for $1 \mathrm{~h}$ at room temperature with the appropriate secondary biotinylated goat anti-mouse or anti-rabbit immunoglobulin $\mathrm{G}$ (1:200; cat. nos. BA9200 and BA1000; Vector Laboratories) and with peroxidase-conjugated avidin (Vectastain Elite ABC kit standard* PK 6-100; Vector Laboratories) for $35 \mathrm{~min}$. Following a further wash with PBS, slides were treated with 0,05\% 3,3-diaminobenzidine (DAB) and 0,1\% $\mathrm{H}_{2} \mathrm{O}_{2}$ (DAB substrate kit for peroxidase, Vector Laboratories; SK-4100). Finally, sections were counter-stained with Mayer's hematoxylin and observed using a light microscope (Axio Lab.A1; Zeiss, Oberkochen, Germany). Negative control experiments were performed by omitting the primary antibody, substituting the primary antibody with an equivalent quantity of non-specific immunoglobulins or pre-incubating the primary antibody with the specific blocking peptide (antigen/antibody=5 according to the manufacturer's instructions). The staining assessment was made by two observers experienced in light microscopy. Immunoreactivity was assessed for IL-1 $\beta$, IL-6, TNF- $\alpha$, VEGF, ICAM-1 and TGF- $\beta 1$ in Schwann cells, vascular endothelium and dense connective tissue of neoplastic vestibular nerve samples, and compared with that of the healthy samples. The intensity of the immune reaction was assessed microdensito- metrically using an IAS 2000 image analyzer (Delta Sistemi, Rome, Italy) connected via a TV camera to the microscope. The system was calibrated using zero as the background obtained in sections exposed to non-immune serum. A total of ten $100-\mu \mathrm{m}^{2}$ areas were delineated in each section using a measuring diaphragm. The quantitative data regarding the intensity of immune staining were analyzed statistically using an analysis of variance followed by Duncan's multiple range test as a post hoc test.

Statistical analysis. The comparison of the expression levels of TNF- $\alpha$, TGF- $\beta 1$, IL-1 $\beta$, IL- 6 and VEGF between the VS and normal vestibular nerve samples was performed using a t-test. Statistical analyses were performed using the SPSS statistical software package version 12.0 (SPSS, Inc., Chicago, IL, USA). $\mathrm{P}<0.001$ was considered to indicate a statistically significant difference.

\section{Results}

Morphological analysis of vestibular nerves using $H \& E$ staining. A total of 10 patients, ranging in age between 45 and 69 years, with a diagnosis of VS were investigated. A total of 10 samples of healthy vestibular nerves were obtained via vestibular neurectomy to serve as controls. The control peripheral nerve samples consisted of a single fascicle surrounded by a dense perineurium, containing small vessels (Fig. 1). The greatest number of nuclei within the fascicle were attributed to Schwann cells. The shape and the arrangement of these nuclei reflected the course of individual axons. The fibroblasts of the endoneurium were dispersed amongst numerous Schwann cells, and exhibited thinner nuclei and increased cellular condensation compared with the Schwann cells. The nuclei of Schwann cells were elongated along the major axis of the nerve (Fig. 1A). Sections of VS samples exhibited compact spindle cell areas, which were densely populated, and formed a fascicular, storiform and whorled-growth pattern. Histologically, schwannomas are composed of spindle cells arranged in bundles with elongated nuclei that form Verocay's bodies (Fig. 1B). Phenomena associated with cystic degeneration are common. Thickening and hyalinization of the vessel walls are associated with microhemorrhagic phenomena. The sections were exposed to primary and secondary antibodies, resulting in the development of dark-brown (intense), yellow-brown (slight) or no immune staining. Immunoreactivity was deemed to be specific as no immunostaining was observed in control sections incubated with primary antibodies absorbed with the specific peptide or with the pre-immune serum.

Immunohistochemical analysis of human healthy vestibular nerve and schwannoma samples. All VS samples examined in the present study exhibited a marked immunoreactivity for TGF- $\beta 1$. TGF- $\beta 1$ immunoreactivity was detected primarily in the cytoplasm of Schwann cells and revealed differences in the number of immunopositive cells between Antoni A and Antoni B tissue types. Antoni A and Antoni B tissue types represent distinct histologic architectural patterns that aid in the histopathologic diagnosis of schwannoma (21). Type A tissue is highly cellular and demonstrates nuclear palisading as well as associated Verocay bodies, which reflects their 


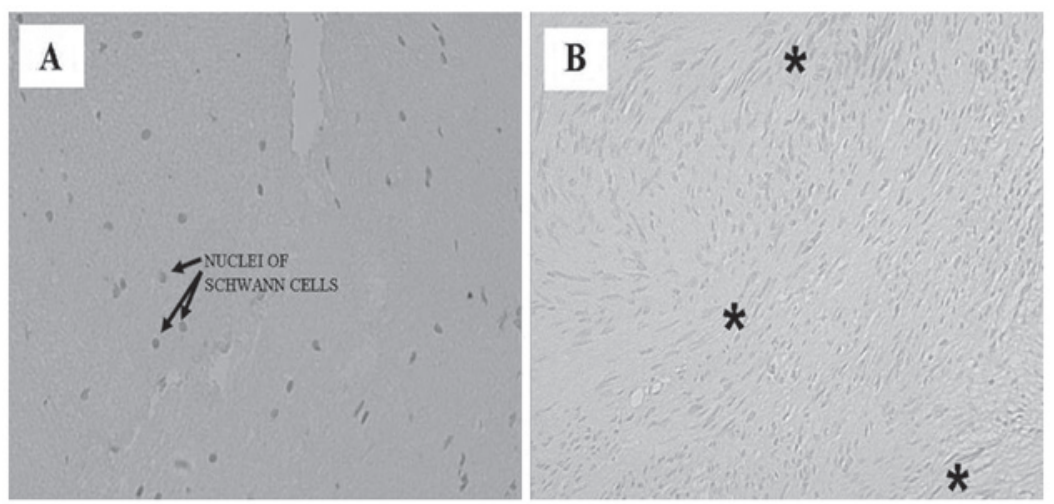

Figure 1. H\&E staining of human healthy vestibular nerve and schwannoma samples. (A) H\&E staining of a control nerve section revealed nuclei of Schwann cells in the endoneurium, which also contained several fibroblasts. (B) H\&E staining of vestibular schwannoma section that exhibited Verocay Bodies (Antoni A regions), consisting of spindle cells arranged in bundles with elongated nuclei (indicated by *). Magnification, x20. H\&E, hematoxylin and eosin.
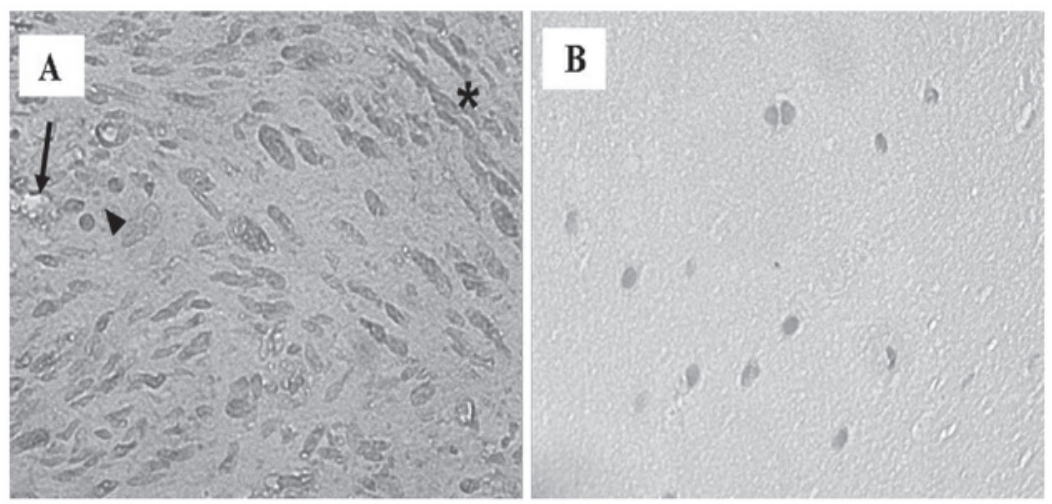

Figure 2. Immunohistochemical analysis of human healthy vestibular nerve and schwannoma samples for TGF- $\beta 1$. (A) TGF- $\beta 1$ expression in vestibular schwannoma tissue section revealed a cytoplasmic localization in Schwann cells. Immunoreactivity was increased in Antoni A areas (*) compared with Antoni B regions (४). TGF- $\beta 1$ reactivity was also observed in the endothelial cells of the blood vessels distributed in the endoneurium $(\rightarrow)$. (B) Immunoreactivity for TGF- $\beta 1$ was not detected in the control samples. Magnification, $\mathrm{x} 40$. TGF- $\beta 1$, transforming growth factor- $\beta 1$.

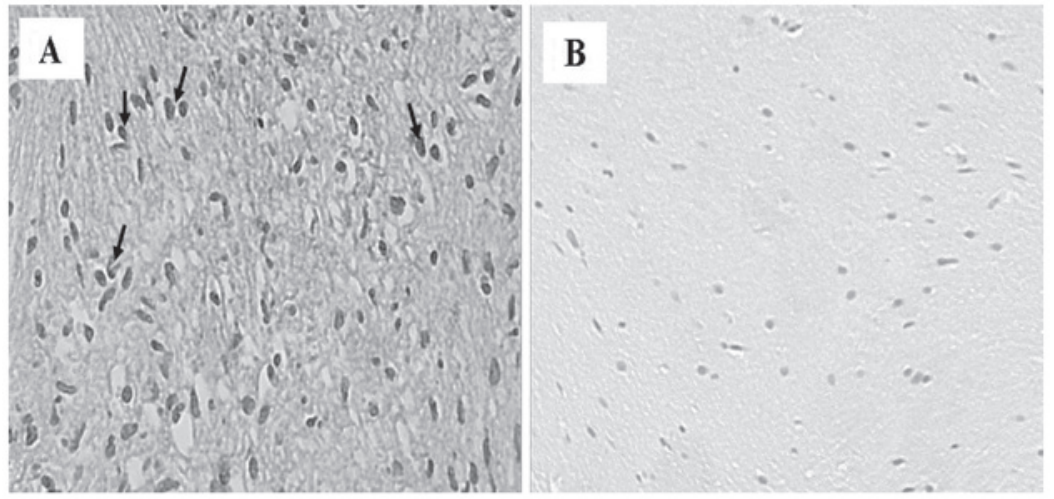

Figure 3. Immunohistochemical analysis of human healthy vestibular nerve and schwannoma samples for TNF- $\alpha$. (A) TNF- $\alpha$ immunoreactivity in vestibular schwannoma tissue section revealed a weak expression in the blood vessel wall and in Antoni A and B regions. In addition, Antoni A regions exhibited the same immunoreactivity as that in the nuclei of Schwann cells $(\rightarrow)$. (B) Absence of TNF- $\alpha$ expression in the control samples was observed. Magnification, $\mathrm{x} 20$ TNF- $\alpha$, tumor necrosis factor- $\alpha$.

prominent extracellular matrix and secretion of laminin. Type B tissue is loosely organized with myxomatous and cystic change and may represent degenerated Antoni A tissue. Antoni A cellular areas expressed more prominent TGF- $\beta 1$ immunoreactivity than Antoni B areas. The Antoni B regions exhibited less dense cellular areas than Antoni A regions, in which there were compactly arranged spindle cells with long and oval nuclei (Fig. 2A). TGF- $\beta 1$ reactivity was also demonstrated in the blood vessel walls distributed in the neoplastic dense connective tissue. No immunoreactivity for TGF- $\beta 1$ was observed in vascular endothelial cells, or Antoni A and Antoni B areas in the control specimens (Fig. 2B). TNF- $\alpha$ was 

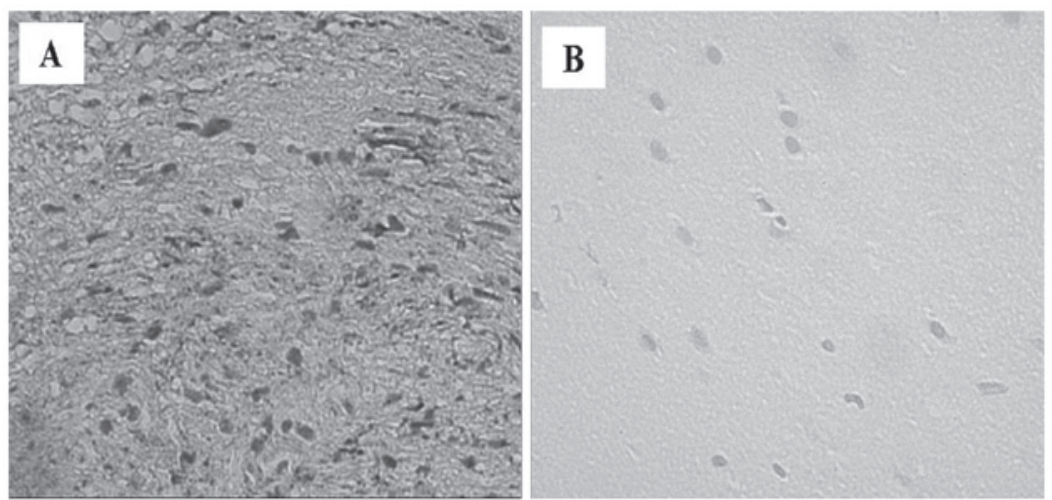

Figure 4. Immunohistochemical analysis of human healthy vestibular nerve and schwannoma samples for IL-1ß. (A) IL-1 $\beta$ immunoreactivity in vestibular schwannoma tissue section revealed marked expression in the Antoni A and B regions. This expression was localized in the cytoplasm of Schwann cells. Moderate immunoreactivity was observed in vascular endothelium. (B) No immunoreactivity for IL-1 $\beta$ in Schwann cells and blood vessels was detected in the control samples. Magnification, x40. IL-1 $\beta$, interleukin-1 $\beta$.
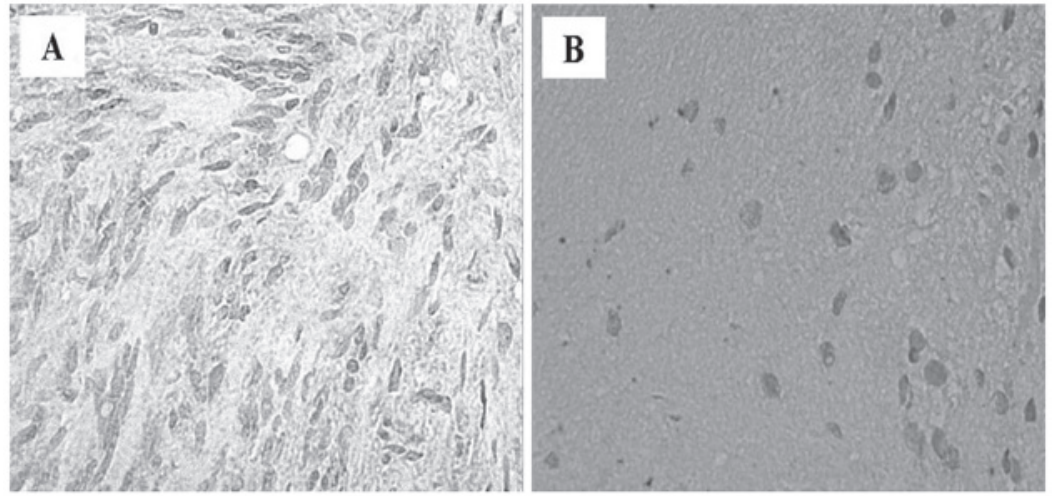

Figure 5. Immunohistochemical analysis of human healthy vestibular nerve and schwannoma samples for IL-6. (A) IL-6 immunoreactivity in vestibular schwannoma tissue section revealed weak cytoplasmic expression in Schwann cells. (B) No immunoreactivity for IL-6 in the cytoplasm of Schwann cells was detected in the control samples. Magnification, x40. IL-6, interleukin-6.

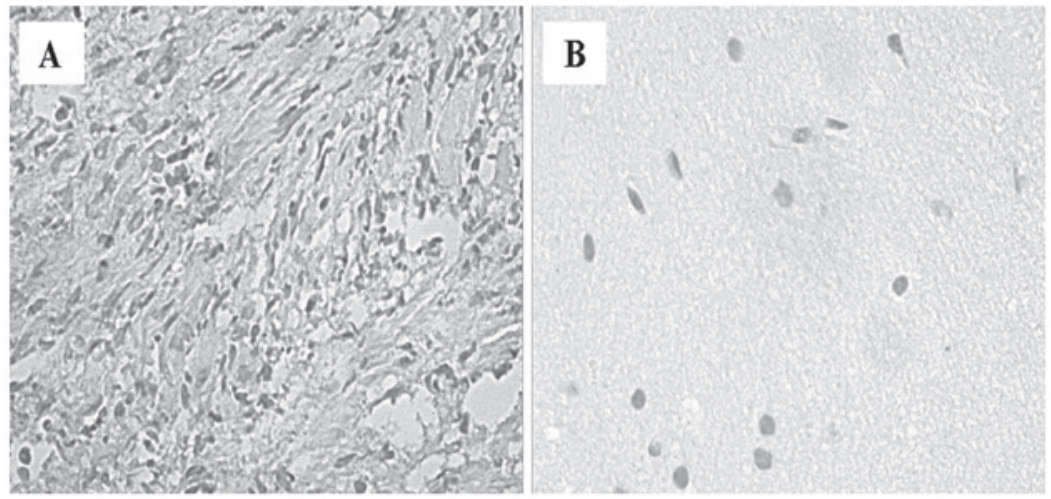

Figure 6. Immunohistochemical analysis of human healthy vestibular nerve and schwannoma samples for ICAM-1. (A) Immunoreactivity for ICAM-1 in vestibular schwannoma tissue sections revealed marked expression in the cytoplasm of Schwann cells. (B) No immunoreactivity for ICAM-1 was observed in the control samples. Magnification, x40. ICAM-1, intracellular adhesion molecule-1.

weakly expressed by the majority of human VS specimens in the endothelial cells of vessels, and Antoni A and Antoni B regions. Numerous immunopositive nuclei were detected in the Antoni A regions compared with the Antoni B regions (Fig. 3A). No immunoreactivity for TNF- $\alpha$ was detected in the healthy control vestibular nerve samples (Fig. 3B). There was increased expression of IL-1 $\beta$ within the VS tissues (Fig. 4A), which was localized to the cytoplasm of Schwann cells. Antoni A and $\mathrm{B}$ regions exhibited approximately the same number of immunopositive nuclei for IL-1 $\beta$. A moderate expression for IL-1 $\beta$ was also observed in the blood vessels. No staining was observed in control tissues (Fig. 4B). Immunohistochemistry for IL-6 was positive in the VS cells, with weak expression in the cytoplasm (Fig. 5A), while in the control nerves samples 
Table I. Levels of cytokines and growth factors in vestibular schwannoma tissue samples and in healthy vestibular nerve samples, and corresponding statistical significance (t-test).

\begin{tabular}{|c|c|c|c|}
\hline Factor & $\begin{array}{c}\text { Vestibular } \\
\text { Schwannoma: } \\
10 \text { patients; } 1 \\
\text { specimen for } \\
\text { each patient }(\%)\end{array}$ & $\begin{array}{l}\text { Control samples: } \\
10 \text { patients; } 1 \\
\text { specimen for each } \\
\text { patient }(\%)\end{array}$ & P-value \\
\hline TGF- $\beta 1$ & $84.3 \pm 4.83$ & $2.0 \pm 0.66$ & $<0.0001$ \\
\hline TNF- $\alpha$ & $72.4 \pm 5.14$ & $5.7 \pm 1.33$ & $<0.0001$ \\
\hline IL-1 $\beta$ & $74.1 \pm 6.11$ & $6.0 \pm 1.24$ & $<0.0001$ \\
\hline IL-6 & $63.3 \pm 4.52$ & $2.1 \pm 0.73$ & $<0.0001$ \\
\hline ICAM-1 & $90.4 \pm 4.97$ & $6.1 \pm 1.19$ & $<0.0001$ \\
\hline VEGF & $77.5 \pm 3.30$ & $7.2 \pm 1.03$ & $<0.0001$ \\
\hline
\end{tabular}

Data are presented as the mean \pm standard deviation. TGF- $\beta 1$, transforming growth factor- $\beta 1$; TNF- $\alpha$, tumor necrosis factor- $\alpha$; IL, interleukin; ICAM-1, intracellular adhesion molecule-1; VEGF, vascular endothelial growth factor.

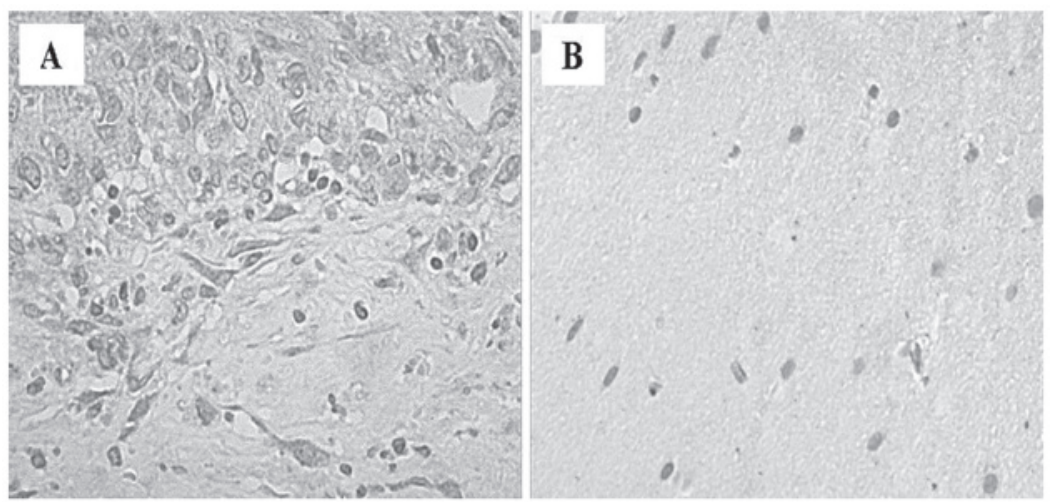

Figure 7. Immunohistochemical analysis for VEGF in human normal vestibular nerve and schwannoma samples. (A) VEGF immunoreactivity was observed as a marked granulated cytoplasmic stain in Schwann cells obtained from the vestibular schwannoma tissue sections. Positive staining was also detected in vascular endothelium of neoplastic tissue. (B) VEGF immunoreactivity was absent in the control samples. Magnification, x40. VEGF, vascular endothelial growth factor.

it was undetectable (Fig. 5B). The findings confirmed that these cytokines are involved in the development and progression of VS via stimulation of Schwann cell proliferation. Pro-inflammatory cytokines, including TGF- $\beta 1, \mathrm{TNF}-\alpha$, IL-1 $\beta$ and IL-6, may be secreted by activated leukocytes, fibroblasts and Schwann cells. These cytokines are known to induce the expression of adhesion molecules, such us ICAM-1 and VCAM-1, and are able to facilitate leucocyte attachment and interactions with cells from the trigger tissue, an area of the neuron that contains a high membrane concentration of voltage-gated Na+ channels. A significantly higher ICAM-1 expression was observed in the cytoplasm of Schwann cells (Fig. 6A), compared with the control nerve samples, in which no staining was identified (Fig. 6B).

Immunohistochemical staining for VEGF was positive in 9/10 of the VS cases (Fig. 7A). A positive expression of VEGF was observed in the tumor samples. VEGF exhibited finely granular cytoplasmic staining in the Schwann cells with intensified focal staining in the perinuclear region. There was only a small variation of staining within the tissues of individual tumors. Staining for VEGF also occurred in the cytoplasm of

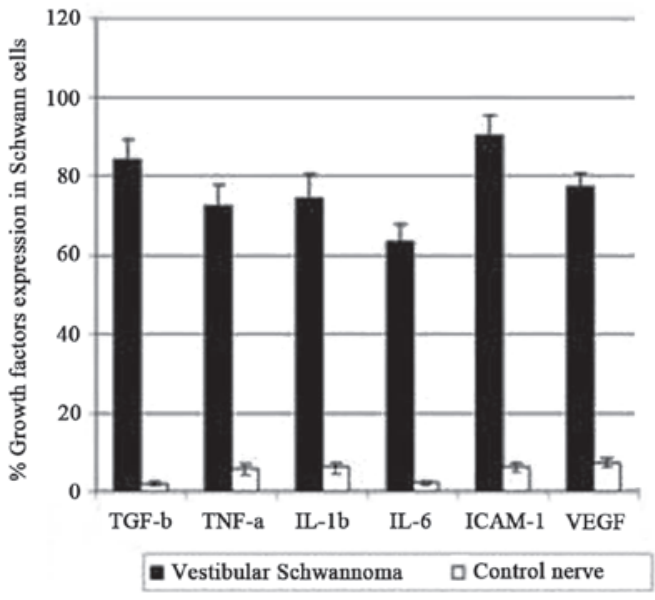

Figure 8. Histogram indicating the percentage of growth factor expression in the vestibular schwannoma cells compared with control tissue. The data underline the differences between the expression of these factors in the two different specimens: Black=schwannoma samples; white=control samples. All differences between the vestibular schwannoma and control samples were statistically significantly $(\mathrm{P}<0.001)$. TGF-1, transforming growth factor-1; TNF- $\alpha$, tumor necrosis factor- $\alpha$; IL, interleukin; ICAM-1, intracellular adhesion molecule-1; VEGF, vascular endothelial growth factor. 
endothelial cells and in polymorphonuclear leukocytes within vessels. These data confirm that VEGF may have a significant impact on the growth VSs, stimulating the mitogenic activity of Schwann cells and angiogenesis in these tumors. VEGF immunoreactivity was absent in the control nerve samples (Fig. 7B).

Statistical analysis of growth factors and cytokines expression. The intensity of staining for TGF- $\beta 1$, TNF- $\alpha$, IL- $1 \beta$, IL-6 and VEGF in human VS and control nerve samples is shown in Table I. The percentage values of pro-inflammatory cytokine-positive cells and P-values are also shown in Fig. 8.

\section{Discussion}

VSs are rare and slow-growing neoplasms, which occur as a result of increased proliferation of Schwann cells of the vestibular branch of the eighth cranial nerve. The tumors generally originate near the myelin-glial junction, close to the internal auditory canal (22). Neoplastic growth appears to rely upon cytokines, which possess angiogenic and mitogenic properties. Limited data concerning the expression of growth factors and its implication on VS growth are available. The aim of the present study was to investigate the role of certain pro-inflammatory cytokines in sporadic VS, associated with angiogenesis and tumor growth. The mechanisms underlying schwannoma development, growth and growth arrest remain to be elucidated. In order to develop an improved understanding of the mechanisms responsible for the growth of these neoplasms, associations between an abnormal proliferation of Schwann cells and the expression of certain inflammatory cytokines were investigated.

Although VSs are relatively slow-growing neoplasms, their continued growth depends on a functional vascular system, as with any other tumor (23). The positive expression of VEGF in VS specimens suggests that angiogenesis is involved in facilitating the growth of this tumor. Indeed, angiogenesis is a prerequisite for the proliferation and progression of a number of neoplasms (23). Despite the evidence suggesting that VS are generally slow-growing tumors, and therefore do not require excessive vascularization, the presence of a functional vascular system remains paramount for tumor development. The results of the present study have revealed a marked expression of VEGF in the cytoplasm of Schwann cells and in vascular endothelial cells from neoplastic peripheral nerves. The present findings confirm that VEGF expression may be involved in the development and expansion of benign tumors as well as malignant ones. In addition to its role as an angiogenic factor, VEGF also possesses neurotrophic and neuroprotective properties in the peripheral and central nervous system, which exert a direct action not only on neurons, but also on Schwann cells (24). The mechanisms responsible for an inflammatory reaction in VS required further elucidation. Inflammation contributes to tumor progression by stimulating the angiogenic process and providing neoplastic cells with growth factors.

VEGF and TGF- $\beta 1$ have are putative key mediators of VS growth (25). Overexpression of TGF- $\beta 1$ increases the invasiveness of neoplastic cells by increasing their proteolytic activity and promoting their binding to cell-adhesion molecules (26). Previous animal studies have identified
TGF- $\beta 1$ as a potent mitogen for Schwann cells. It has also previously been reported that Schwann cells secrete and activate the latent form of TGF- $\beta 1$ (27), thereby stimulating the proliferation of Schwann cells. TGF- $\beta 1$ has been hypothesized to be involved in the regulation of peripheral nerve tumors by modulating cell proliferation and differentiation, by different mechanisms from those of glial growth factors and fibroblast growth factor, which are responsible for the mitogenic activity of Schwann cells $(28,29)$. The results of the present study suggested that TGF- $\beta 1$ may be affect tumor progression by indirectly stimulating angiogenesis through the upregulation of VEGF expression in VS. Cytokines are the primary mediators of communication between cells in the inflammatory tumor micro-environment. It has been established that neoplastic cells express pro-inflammatory mediators, including cytokines, such as TGF- $\beta 1$, IL- 6 , IL- $1 \beta$ and TNF- $\alpha$ (30). TNF- $\alpha$ is known to be a major mediators of inflammation; in addition, $\mathrm{TNF}-\alpha$ was reported to be produced by tumors and to function as an endogenous tumor promoter. TNF- $\alpha$ has been associated with numerous processes involved in tumorigenesis, including cellular transformation, promotion, survival, proliferation, invasion, angiogenesis and metastasis (31). TNF- $\alpha$ upregulates ICAM-1 on the Schwann cell surface, suggesting that these cells also carry functional TNF- $\alpha$ receptors (32). While TNF- $\alpha$ is toxic to numerous types of cell, it is not injurious to cultured Schwann cells (33). However, it does inhibit unstimulated cell proliferation and connexin 46 expression (34). In the present study, it was identified that moderate expression of TNF- $\alpha$ in the cytoplasm of Schwann cells was predominantly localization in Antoni A regions, while this cytokine was completely absent in normal vestibular nerve samples. The cytotoxic or protective effects of TNF- $\alpha$ depend on its receptor, cell type and the presence of other factors. To date, two types of cell surface receptors for TNF- $\alpha$ have been identified: TNFRI (p55) and TNFRII (p75) (35). Activation of TNFRII receptor results in a complex signalling pathway involving numerous other TNF-receptor-activated proteins $(36,37)$. Receptor signaling, via this pathway, triggers the proteolysis of cytoplasmic protein I $\mathrm{B}$, which, in turn, allows translocation of the nuclear transcription factor nuclear factor- $\kappa \mathrm{B}$ (38). This has been observed to lead to apoptosis in specific cell types, whilst it is protective in others (39). In the present study, TNF- $\alpha$ appeared to induce a protective effect in Schwann cells, possibly as a result of the presence on the cellular surface of TNFRII. IL-1 $\beta$, secreted by neoplastic cells or infiltrating leukocytes, is involved in increasing tumor adhesion, invasion, angiogenesis and immune suppression (40). The results of the present study demonstrated that VS is associated with elevated expression of IL-6 and VEGF, indicating that IL- 6 is a possible mediator of the association between VS and systemic inflammatory responses in patients with this disease. Interleukin-6 is involved in peripheral nerve regeneration (41). However, the molecular mechanisms underlying IL-6 function in Schwann cell physiology are yet to be elucidated. The induction of proinflammatory genes by IL-6 in Schwann cells may indicate that IL-6 is involved in the degeneration of the injured nerve, in association with other inflammatory cytokines, including TNF- $\alpha$. IL- 6 may facilitate the demyelination of peripheral nerves following 
nerve injury, and appears to induce degenerative changes in Schwann cells following nerve injury and to activate proinflammatory signals in Schwann cells (42). The majority of IL-6 target genes are involved in cell cycle progression and in the suppression of apoptosis, which emphasizes the importance of IL-6 in tumorigenesis (43). Accordingly, cytokines, including TNF- $\alpha$ and IL-1 $\beta$, are emerging as putative targets for anticancer therapies (44). Specific inhibition of pro-inflammatory mediators, including TNF- $\alpha$, IL- 6 , TGF- $\beta 1$ and IL-1 $\beta$, may lead to a reduction in tumor development and inhibition of transcription associated with the inflammatory process.

TNF- $\alpha$, IL-6, TGF- $\beta 1$ and IL-1 $\beta$ have been shown to upregulate adhesion molecules, such as ICAM-1, in human Schwann cells. In the present study, ICAM-1 was observed to be highly expressed in VS samples and its expression was associated with tumor size and the inflammatory process. Cellular immunity against tumor cells requires the presence of adhesion molecules, such as ICAM-1 on the endothelial surface, which mediate the arrest of leukocytes (16). The induction of the expression of ICAM-1 in Schwann cells by pro-inflammatory cytokines suggests a possible role for adhesion molecules in the pathogenesis of inflammation in the peripheral nerve. The absence of immunoreactivity observed in the control specimens indicates that the hyperexpression of growth factors and cytokines is associated with tumor development or inflammatory conditions.

The current findings suggested that chronic inflammation, through its promotion of angiogenesis, is involved in tumor progression. Angiogenesis appears to be important for the induction of growth of VS as well as the growth of other neoplasms, including glioblastoma in children (45) and primary or metastatic breast cancer (46). Numerous previous studies have reported a significant correlation between the concentration of VEGF and VEGFR-1 expression in VS, and tumor growth rate, but did not describe symptom duration or tumor size (47-49). The present findings confirmed the expression of VEGF, with cytoplasmic localization, in VS samples. Previous experiments in nude mice injected with malignant tumor cells have demonstrated that intravenous infusion of anti-VEGF monoclonal antibodies reduces the growth of tumors by up to $96 \%$ (50). The anti-VEGF therapy was directed toward the suppression of VEGF or its receptors. This treatment, based on the inhibition of VEGF and its receptors, may be a potential option with which to counteract the development of VS.

Additional studies on the involvement of chronic inflammatory processes in the development of VS are required. The first stage may be to further identify the inflammatory cells present in VS. Furthermore, their activation and association with angiogenic growth factors should be examined. VS cells produce and secrete pro-inflammatory cytokines, which may act in an autocrine manner, stimulating cellular proliferation.

The potential use of novel therapeutic approaches based on the combined administration of inhibitors of proinflammatory cytokines and VEGF may hold promise for the development of therapies for neoplastic diseases involving the peripheral nervous system. In conclusion, the present study demonstrated that the development of synthetic inhibitors of growth factors may potentially reduce the recurrence rate of VS and enable non-surgical management of this disease. Additional clinical and experimental investigations are necessary to clarify the biological role of these molecules in the development and progression of this type of neoplasm.

\section{References}

1. McDonald R: Acoustic neuroma: what the evidence says about evaluation and treatment. J Fam Pract 60: E1-E4, 2011.

2. Celis-Aguilar E, Lassalletta L, Torres Martin M, Rodrigues FY, Nistal M, Castresana JS, et al: The molecular biology of vestibular schwannomas and its association with hearing loss: a review. Genet Res Int 2012: 856157, 2012.

3. Bondi S, Limardo P, Toma S and Bussi M: Non-vestibular head and neck schwannomas: a 10-year experience. Eur Arch Otorhinolaryngol 270: 2365-2369, 2013.

4. van Leeuwen JP, Cremers CW, Thewissen NP, Harhangi BS and Meijer E: Acoustic neuroma: correlation among tumor size, symptoms, and patient age. Laryngoscope 105: 701-707, 1995.

5. Carroll SL: Molecular mechanisms promoting the pathogenesis of Schwann cell neoplasms. Acta Neuropathol 123: 321-348, 2012.

6. Klenke C, Widera D, Sepehrnia A, Moffat DA, Kaltschmidt C, Kaltschmidt B, Ebmeyer J and Sudhoff H: Clinical and biological behaviour of vestibular schwannomas: signalling cascades involved in vestibular Schwannoma resemble molecular and cellular mechanisms of injury-induced Schwann cell dedifferentiation. Head Neck Oncol 16: 20, 2013.

7. Mirsky R, Parmantier E, McMahon AP and Jessen KR: Schwann cell-derived desert Hedgehog signals nerve sheath formation. Ann NY Acad Sci 883: 196-202, 1999.

8. Chen ZL and Strickland S: Laminin gamma1 is critical for Schwann cell differentiation, axon myelination, and regeneration in the peripheral nerve. J Cell Biol 163: 889-899, 2003.

9. Weerda H, Gamberger TI, Siegner A, Gjuric M and Tamm ER: Effects of transforming growth factor- $\beta 1$ and basic fibroblast growth factor on proliferation of cell cultures derived from human vestibular nerve schwannoma. Acta Otolaryngol 118: 337-343, 1998.

10. Diensthuber M, Brandis A, Lenarz T and Stover T: Co-expression of transforming growth factor- $\beta 1$ and glial cell line-derived neurotrophic factor in vestibular schwannoma, Otol Neurotol 25: 359-365, 2004.

11. Löttrich M, Mawrin C, Chamaon K, Kirches E, Dietzmann K, and Freigang B: Expression of transforming growth factor-beta receptor type 1 and type 2 in human sporadic vestibular Schwannoma. Pathol Res Pract 203: 245-249, 2007.

12. Wagner R and Myers RR: Schwann cells produce tumor necrosis factor alpha: expression in injured and non-injured nerves. Neuroscience 73: 625-629, 1996.

13. Wang Y, Tang X, Yu B, Gu Y, Yuan Y, Yao D, Ding F and Gu X: Gene network revealed involvements of Birc2, Birc3 and Tnfrsfla in anti-apoptosis of injured peripheral nerves. PloS One 7: e43436, 2012.

14. Lee HK, Seo IA, Suh DJ, Hong JI, Yoo YH and Park HT: Interleukin-6 is required for the early induction of glial fibrillary acidic protein in Schwann cells during Wallerian degeneration. J Neurochem 108: 776-786, 2009.

15. Bolin LM, Verity AN, Silver JE, Shooter EM and Abrams JS: Interleukin- 6 production by Schwann cells and induction in sciatic nerve injury. J Neurochem 64: 850-858, 1995.

16. Constantin G, Piccio L, Bussini S, Pizzuti A, Scarpini E, Baron P, Conti G, Pizzul S and Scarlato G: Induction of adhesion molecules on human Schwann cells by proinflammatory cytokines, an immunofluorescence study. J Neurol Sci 170: 124-130, 1999.

17. Bevilacqua MP: Endothelial-leukocyte adhesion molecules. Annu Rev Immunol 11: 767-804, 1993.

18. Møller MN, Werther K, Nalla A, Stangerup SE, Thomsen J, Bøg-Hansen TC, Nielsen HJ and Cayé-Thomasen P: Angiogenesis in vestibular schwannomas: expression of extracellular matrix factors MMP-2, MMP-9 and TIMP-1. Laryngoscope 120: 657-662, 2010.

19. Ferrara N, Gerber HP and LeCouter J: The biology of VEGF and its receptors. Nat Med 9: 669-676, 2003.

20. Bancroft JD and Gamble M (eds): Theory and Practice of Histological Techniques. $6^{\text {th }}$ edition. Churchill Livingstone Elsevier, London, 2008.

21. Joshi R: Learning from eponyms: Jose Verocay and Verocay bodies, Antoni A and B areas, Nils Antoni and Schwannomas. Indian Dermatol Online J. 3: 215-219, 2012. 
22. Charabi S: Acoustic neuroma/vestibular schwannoma in vivo and in vitro growth models. A clinical and experimental study. Acta Otolaryngol Suppl 530: 1-27, 1997.

23. Folkman J: Tumor angiogenesis: therapeutic implications. NEngl J Med 285: 1182-1186, 1971.

24. Namiecińska M, Marciniak K and Nowak JZ: VEGF as an angiogenic neurotrophic, and neuroprotective factor. Postepy Hig Med Dosw (Online) 59: 573-583, 2005 (In Polish).

25. Kramer F, Stöver T, Wamecke A, Diensthuber M, Lenarz T and Wissel K: BDNF mRNA expression is significantly upregulated in vestibular schwannomas and correlates with proliferative activity. J Neurooncol 98: 31-39, 2010.

26. Maehara Y, Kakeji Y, Kabashima A, Emi Y, Watanabe A, Alkazawa K, Baba H, Kohnoe S and Sugimachi K: Role of transforming growth factor-beta 1 in invasion and metastasis in gastric carcinoma. J Clin Oncol 17: 607-614, 1999.

27. Ridley AJ, Davis JB, Stroobant P and Land H: Transforming growth factors-beta 1 and beta 2 are mitogens for rat Schwann cells. J Cell Biol 109: 3419-3424, 1989.

28. Lemke GE and Brockes JP: Identification and purification of glial growth factor. J Neurosci 4: 74-83, 1984.

29. Ratner N, Bunge RP and Glaser L: Schwann cell proliferation in vitro. An overview. Ann NY Acad Sci 486: 170-181, 1986.

30. Candido J and Hagemann T: Cancer-related inflammation. J Clin Immunol 33: S79-S84, 2013

31. Sethi G, Sung B and Aggaewal BB: TNF: a master switch for inflammation to cancer. Front Biosci 13: 5094-5107, 2008.

32. Lisak RP and Bealmear B: Upregulation of intercellular adhesion molecule-1 (ICAM-1) on rat Schwann cells in vitro: comparison of interferon-gamma, tumor necrosis factor-alpha and interleukin-1. J Peripher Nerv Syst 2: 233-243, 1997.

33. Mithen F, Colburn S and Birchem R: Human alpha tumor necrosis factor does not damage cultures containing rat Schwann cells and sensory neurons. Neurosci Res 9: 59-63, 1990.

34. Chandross KJ, Spray DC, Cohen RI, Kumar NM, Kremer M, Dermietzel R and Kessler JA: TNF-alpha inhibits Schwann cell proliferation, connexin46 expression and gap junctional communication. Mol Cell Neurosci 7: 479-500, 1996.

35. Uren AG and Vaux DL: Molecular and clinical aspects of apoptosis. Pharmacol Ther 72: 37-50, 1996

36. Nagano K, Alles N, Mian AH, et al: The tumor necrosis factor type 2 receptor plays a protective role in tumor necrosis factor- $\alpha$-induced bone resorption lacunae on mouse calvariae. J Bone Miner Metab 29: 671-681, 2011.
37. Tracey D, Klareskog L, Sasso EH, Salfeld JG and Tak PP: Tumor necrosis factor antagonist mechanism of action: a comprehensive review. Pharmacol Ther 117: 244-279, 2008

38. Baker SJ and Reddy EP: Transducers of life and death: TNF receptor superfamily and associated proteins. Oncogene 12 : $1-9,1996$.

39. Beg AA and Baltimore D: An essential role for NF-kappaB in preventing TNF-alpha-induced cell death. Science 274 782-784, 1996

40. Colotta F, Allavena P, Sica A, Garlanda C and Mantovani A: Cancer-related inflammation, the seventh hallmark of cancer: links to genetic instability. Carcinogenesis 30: 1073-1081, 2009.

41. Zhong J, Dietzel ID, Wahle P, Kopf M and Heumann R: Sensory impairments and delayed regeneration of sensory axons in interleukin-6-deficient mice. J Neurosci 19: 4305-4313, 1999.

42. Lee HK, Wang L, Shin YK, Lee KY, Suh DJ and Park HT: Interleukin-6 induces proinflammatory signaling in Schwann cells: a high-throughput analysis. Biochem Biophy Res Commun 382: 410-414, 2009.

43. Haura EB, Turkson J and Jove R: Mechanisms of disease: insights into the emerging role of signal transducers and activators of transcription in cancer. Nat Clin Pract Oncol 2: 315-324, 2005.

44. Klampfer L: Cytokines, inflammation and colon cancer. Curr Cancer Drug Targets 11: 451-464, 2011.

45. Artico M, Cervoni L, Celli P, Salvati M and Palma L: Supratentorial glioblastoma in children a series of 27 surgically treated cases. Childs Nerv Syst 9: 7-9, 1993.

46. Gobbi G, Mirandola P, Micheloni C, et al: Expression of HLA class I antigen and proteasome subunits LMP-2 and LMP-10 in primary vs. metastatic breast carcinoma lesions. Int J Oncol 25: 1625-1629, 2004.

47. Cayé-Thomasen P, Werther K, Nalla A, et al: VEGF and VEGF receptor-1 concentration in vestibular schwannoma homogenates correlates to tumor growth rate. Otol Neurotol 26: 98-101, 2005.

48. Brieger J, Bedavanija A, Lehr HA, Maurer J and Mann WJ: Expression of angiogenic growth factors in acoustic neurinoma. Acta Otolaryngol 123: 1040-1045, 2003.

49. Koutsimpelas D, Bjelopavlovic M, Yetis R, et al: The VEGF/VEGFR axis in sporadic vestibular schwannoma correlates with irradiation and disease recurrence. ORL J Otorinilaryngol Relat Spec 74: 330-338, 2012.

50. Kim KJ, Li B, Winer J, et al: Inhibition of vascular endothelial growth factor-induced angiogenesis suppresses tumour growth in vivo. Nature 362: 841-844, 1993. 